\title{
Studies on the distribution of heavy metal Cd in contaminated soils of various particle sizes and removal efficiencies of heavy metal using acid washing
}

\author{
S. F. Cheng ${ }^{1}$, C. Y. Huang ${ }^{2}$ \& L. S. Hsiao ${ }^{1}$ \\ ${ }^{I}$ Department of Environmental Engineering and Management, \\ Chaoyang University of Technology, Taiwan \\ ${ }^{2}$ Ordnance Readiness Development Center, Taiwan
}

\begin{abstract}
Improper treatment and disposal of industrial wastewater and solid wastes result in serious heavy metal contamination of soil. A commonly used method for remediation of the soil contaminated by heavy metals is acid washing. This method is simple in principle, easy to operate and efficient in achieving the removal of heavy metals.

The experience gained on soil remediation in Taiwan reveals that the soil particle distribution influences the heavy metal removal efficiency to a large extent. Hence, in this research, soil samples were collected from the contaminated sites and used in the investigation on the distribution of heavy metal $\mathrm{Cd}$ in soils of various particle sizes and the efficiencies of acid washing to remove the $\mathrm{Cd}$ from the soil of various particle distributions. The results will be used for future engineering implementation of the acid washing technique.

The research results indicate that soils containing particles with sizes below $0.150 \mathrm{~mm}$ will hold increasing quantities of $\mathrm{Cd}$ and organic matter at decreasing particles sizes. As far as the acid washing efficiency is concerned, smaller particle diameters and higher organic matter contents result in decreasing efficiencies.
\end{abstract}

Keywords: soil, Cd, particle size, CEC, organic matter, acid washing. 


\section{Introduction}

The rapid industrial development in Taiwan during last several decades have not gone together with adequate planning and management of industrial wastewater treatment and disposal. Irrigation channels have often been used to discharge industrial waste effluent thus causing serious pollution to large tracts of farmland. Among all problems, the contamination caused by heavy metals, which are accumulative and non-degradable, is the most serious.

The acid-washing method is commonly used for remediation of heavy metal contaminated soils. This method is simple in principle and easy to implement to come up with good results in a short period of time [1]. It is considered as a preferred method for remediation metal contaminated farmland in Taiwan. However, the experience with several recent cases of soil remediation using the acid-washing method shows that the efficiency depends on the type of soil to a great extent. For sandy loam soil, this method can achieve metal removal efficiency of as high as $90 \%$ while for soils containing fine clay particles, a poor separation of the soil particle and the washing liquid causes a great deal of operational problems. Additionally, the soil that contains fine soil particles exhibits poor permeability thus making it impossible to have effective contacts between the washing liquid and soil particles. Hence, the on-site treatment has removal efficiency lower than $30 \%$; it may sometimes not show any removal efficiency at all.

The literature information [1] shows that most pollutants exist in the portion of soil particles having less than $63 \mu \mathrm{m}$ with relatively larger specific surface area of the contaminated soil. Hence, sieving the soil into two portions prior to the acid treatment is recommended. The larger particles have a lower metal contamination and are considered as clean soil; only the smaller particles need to be washed in the subsequent acid-washing treatment [2]. Anderson et al. [3] pointed out that most of the contaminants concentrate in the portion consisting of silts and clays with less than $50 \mu \mathrm{m}$ diameters. The volume of this portion is a small percentage of the overall soil volume. Thus, the prior sieving procedure may reduce $60 \sim 80 \%$ volume of the soil to be treated thus reducing the treatment cost. Results obtained by Sheets and Bergquist [4] on PCB and lead contents of soil samples collected at superfund sites indicate that soil particles with diameters less than $74 \mu \mathrm{m}$ constitute about $8 \sim 15 \%$ of the total soil mass. Nevertheless, most PCBs exist in the soil particles with less than $74 \mu \mathrm{m}$ diameters. Smaller particles, which have higher specific surface area and contain more organic substance to enhance the adsorption of contaminants, show higher PCB concentrations. The distribution of heavy metals generally follows the similar trend that smaller particles have higher metal concentrations. Particles with diameters between 0.425 to $2 \mathrm{~mm}$ have much higher metal concentrations. Thus, the presence of small lead particles is regarded as the cause of the observed high metal concentration in this soil particle diameter range. Di Palma and Medici [5] combined sulfuric acid and EDTA as the washing agent to wash copper contaminated soil and reported that soils with higher content of organic 
substances exhibit lower copper removal efficiencies mainly because the complex reactions between copper and the organic substances.

In this research, soil samples collected from the heavy metal contaminated field in Taiwan are used to study particle distribution as well as concentrations of heavy metal $\mathrm{Cd}$ and organic substances in soil particles of various sizes. The objective is to understand the correlation between distribution of heavy metals and soil particle size as well as organic substance contents. Additionally, the efficiency of extracting the metals from the contaminated soils using various acid solutions will be studied and the results will be used as references for future implementation of the acid-washing method.

\section{Materials and methodology}

\subsection{Soil preparation}

Samples used in this research were collected from the top $15 \mathrm{~cm}$ layer of a cadmium-contaminated farmland located at Huwei Township, Yunlin Hsieh in central Taiwan. The collected sample was thoroughly mixed; a portion of it was subject to basic characteristic analyses on $\mathrm{pH}, \mathrm{CEC}$, organic substances, water content and heavy metals. The remaining portion was dried, crushed and screened through a 10-mesh $(2 \mathrm{~mm})$ sieve to remove large pebbles and impurities.

The screened samples were then separated into 4 groups of different particle ranges using the wet screening and the gravitational sedimentation methods. First, aqueous $\left(\mathrm{NaPO}_{3}\right)_{6}$ solution was added to the sample to disperse soil particle. After thorough mixing, the soil solution was passed thorough 100 mesh $(0.150 \mathrm{~mm})$ and then 270 mesh $(0.053 \mathrm{~mm})$ wire screens for separating the particles into "sand 1" with particle diameters between 2 and $0.150 \mathrm{~mm}$ and "sand 2" with particle diameters range of 0.150 to $0.053 \mathrm{~mm}$. The solution that had passed through the wire screens was poured into a $1000 \mathrm{ml}$ sedimentation container. Based on Stock's law, the particles settled at the bottom after 7 hours and 36 minutes are silt with diameters between $0.053 \sim 0.002 \mathrm{~mm}$. The remaining particles suspended in the solution are clay with diameters below $0.002 \mathrm{~mm}$; they were filtered out through $0.20 \mu \mathrm{m}$ and then dried.

\subsection{Soil characteristic analyses}

Both the original and the processed soil samples were analyzed for organic substance and CEC. The organic substance analyses were done using the combustion method by heating 20 grams of the dried soil sample at $500 \sim 600^{\circ} \mathrm{C}$ for 4 hours. The weight loss after heating is taken as the soil organic substance content. For measuring the soil CEC, 4 grams of the dried sample was mixed with sodium acetate solution such allowing $\mathrm{Na}^{+}$ion to replace all exchangeable cations contained in the soil sample. The mixture was then added with ammonium acetate to replace all $\mathrm{Na}^{+}$ions by ammonium ions; the concentration of replaced $\mathrm{Na}^{+}$ions is determined using the induced couple plasma (ICP) method as the soil CEC. 


\subsection{Soil heavy metal analyses}

The dried soil samples were digested with aqua regia by adding $21 \mathrm{ml}$ concentrate hydrochloric acid and $7 \mathrm{ml}$ nitric acid with $3 \mathrm{~g}$ of soil slowly. After mixing, the sample was undisturbed for 16 hours. It was then diluted to $100 \mathrm{ml}$ and filtered through $0.20 \mu \mathrm{m}$ filter paper. The filtrate was analyzed for cadmium using the ICP method.

\subsection{Acid-washing of soil}

Soil samples containing various particle sizes were washed with single acids including two inorganic acids (hydrochloric acid and nitric acid) and one organic acid (citric acid) as well as the mixture of hydrochloric and citric acids to study the efficiencies of heavy metal removal from the acid-washed soil. The acid concentrations were $0.01 \mathrm{M}, 0.05 \mathrm{M}$ and $0.1 \mathrm{M}$; the mixed acid solution was prepared by mixing 1 volume of hydrochloric acid and 1 volume of citric acid to final concentrations of $0.01 \mathrm{M}, 0.05 \mathrm{M}$ and $0.1 \mathrm{M}$.

An aliquot of $10 \mathrm{ml}$ washing solution was added to $1 \mathrm{~g}$ soil sample. After mixed on a 150 rpms vibrating shaker for 10 minutes, the mixture was filtered through $0.20 \mu \mathrm{m}$ filter paper. The washed soil sample was then analyzed for heavy metals to calculate the metal removal efficiency.

All analyses were carried out with triplicate samples and the results were used to calculated average and standard deviations.

\section{Results and discussion}

\subsection{Soil characteristics}

Table 1 lists the results of basic soil characteristics. According to the USDA soil classification standards, the contaminated soil is sandy loam containing $125.2 \sim$ $128.2 \mathrm{mg} / \mathrm{kg}$ cadmium. It has average $22.7 \mathrm{meq} / 100 \mathrm{~g} \mathrm{CEC}$ and contains $5.2 \%$ organic matter.

Table 1: $\quad$ Properties of the Cd-contaminated soils.

\begin{tabular}{lr}
\hline $\mathrm{pH}$ & $6.83(0.09)$ \\
Organic content (by weight)\% & $5.15(0.05)$ \\
Water content (by weight)\% & $2.81(0.06)$ \\
$\mathrm{CEC}$ (meq per 100 g) & $22.7(1.30)$ \\
Sand (by weight)\% & $68.5(1.63)$ \\
Silt (by weight)\% & $27.0(0.21)$ \\
Clay (weight)\% & $5.5(0.46)$ \\
\hline Values in parentheses denote standard deviations $(\mathrm{n}=3)$.
\end{tabular}




\subsection{Soil particle and $\mathrm{Cd}$ distributions}

Figure 1 shows variations of the particle and cadmium distributions. The results indicate that "sand 2" particles; i.e. particle with diameters between $0.150 \mathrm{~mm}$ to $0.053 \mathrm{~mm}$, constitute a major portion of the soil of nearly $48 \%$. Additionally, the cumulative mass of all soil particles with diameter greater than $0.053 \mathrm{~mm}$ is $70 \%$ of the total soil mass. Fig. 1 also indicates that the cadmium content in these $70 \%$ soil particles is much lower than those in the clay and silt portions. The "sand 2" particles have the highest mass percentage but the lowest cadmium content of $48.74 \mathrm{mg} / \mathrm{kg}$. On the contrary, the clay portion has the lowest mass percentage but the highest cadmium concentration of $557.37 \mathrm{~kg} / \mathrm{mg}$ that is 11 time the cadmium concentration contained in "sand 2".

During the acid-washing process, clay and silt cannot be effectively separated from the liquid due to their small particle sizes thus lowering the washing efficiency and raising the treatment costs. Adopting the screening procedure to separate smaller particles that contain higher cadmium concentrations to be treated separated will greatly enhance the remediation efficiency for heavy metal removal.

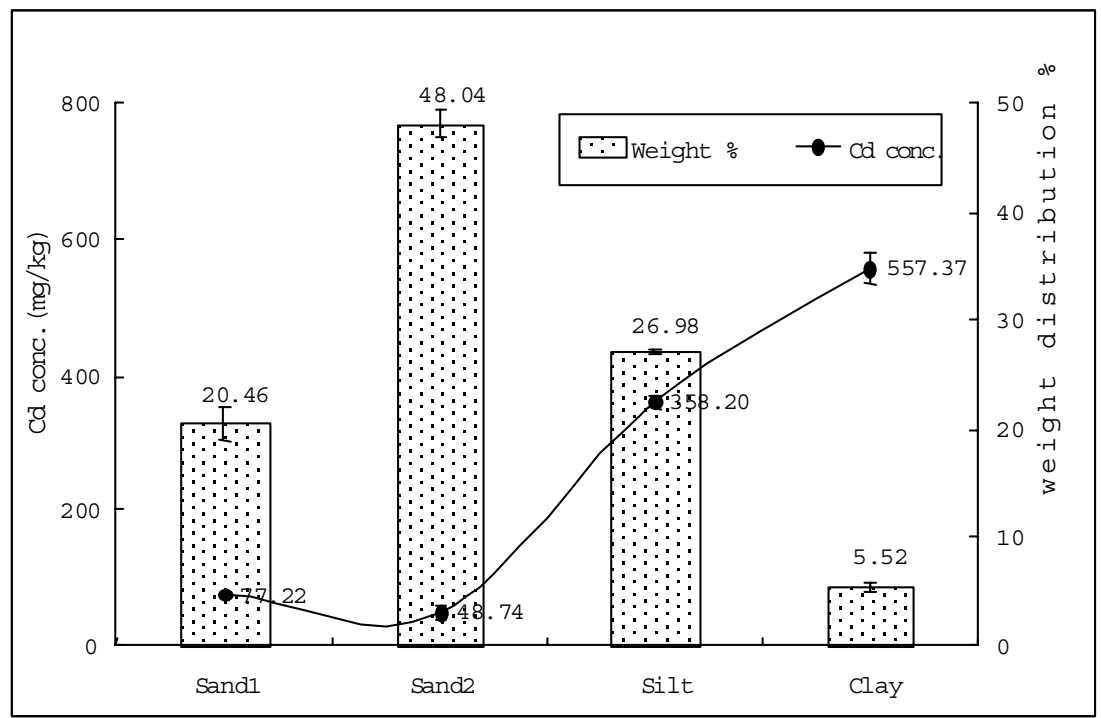

Figure 1: Distribution of particle sizes and $\mathrm{Cd}$ content of various particle sizes for contaminated soil. Error bars are \pm standard deviations for $\mathrm{n}=3$.

\subsection{Correlation between soil characteristics and heavy metal contents}

The correlation between organic matter content and heavy metal concentration for soil samples containing particles of various sizes is shown in Figure 2. For sand 2 to clay, smaller particles contain higher concentrations of organic 
substances. Since the average soil has higher humic substances associated with particles of smaller diameters, the organic content is expected to be higher in soils containing smaller particles. This is contrary to the observation made in this study that "sand 1" soil has the organic content more than "sand 2". However, most of these organic substances are non-decomposed animal and vegetation impurities.

Results shown in Figure 2 indicate similar trends of variations for heavy metal concentration and organic content in soils of various particles. For "sand 2 " to clay, smaller particles contain more organic substances and heavy metals. While "sand 1" shows the increasing organic content is not seen by a proportional increase of cadmium although the cadmium is seen to increase. This observation may be caused by the fact that most organic substances contained in "sand 1" are large non-humic thus they do not have high affinity for heavy metals as those contained in "sand 2".

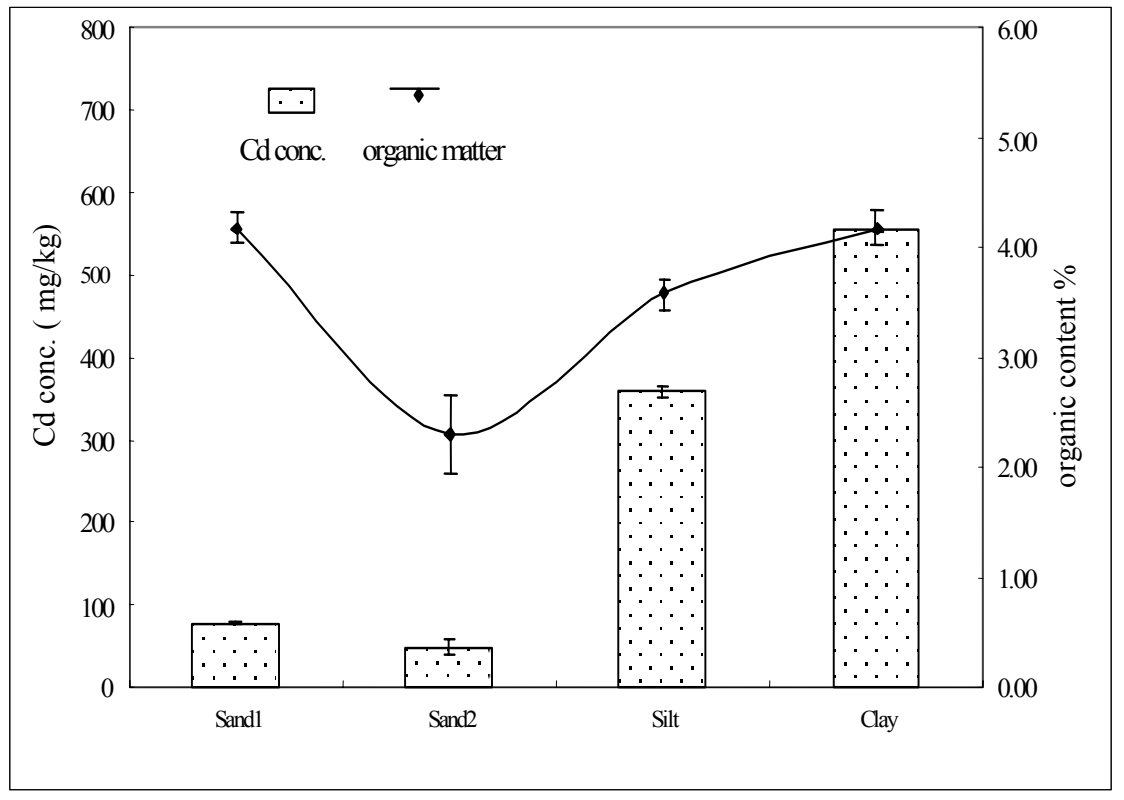

Figure 2: The correlation between organic matter content and heavy metal concentration for soil samples containing particles of various sizes.

The correlation between CEC and heavy metal content for soils containing particles of various sizes as shown in Figure 3 indicates that "sand 1" soil has the lowest CEC. Further, variations of CEC and organic content show similar tendency. In "sand 2" and Clay, higher CECs are associated with small soil particles while "sand 1" has higher CEC than "sand 2". The small increase of CEC in "sand 1" proves that portions of the organic substances contained in "sand 1" are non-decomposed large particles and they do not have much adsorption capacity for heavy metals. 


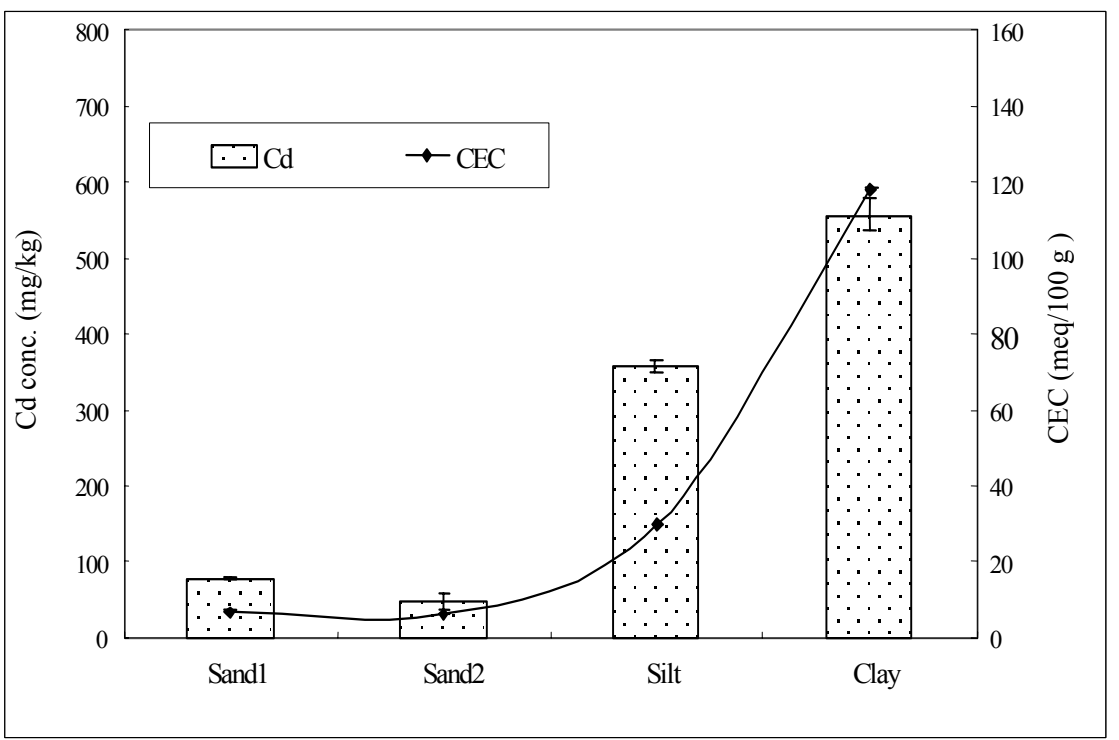

Figure 3: The correlation between CEC and heavy metal content for soils containing particles of various sizes.

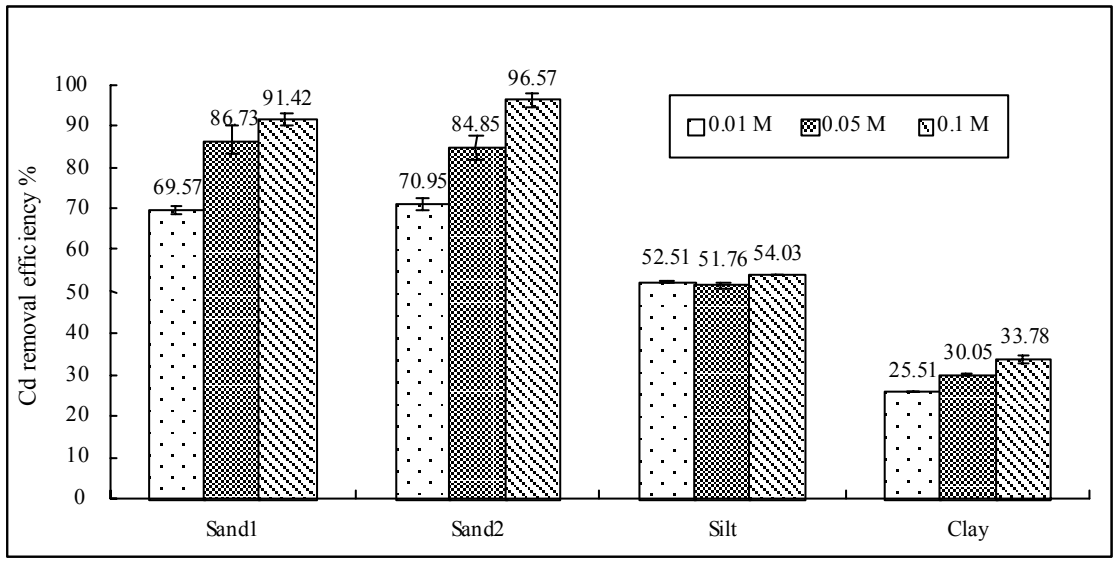

Figure 4: The metal Cd removal efficiencies from soil containing particles of various sizes using hydrochloric acid.

\subsection{Efficiencies for heavy metal removal by acid-washing for particles of various sizes}

The metal Cd removal efficiencies from soil containing particles of various sizes using hydrochloric acid, nitric acid, citric acid and hydrochloric acid/citric acid mixture are shown in Figure 4 to 7 , respectively. Results shown in all figures 
illustrate that the "sand 1" and "sand 2" particles have much higher removal efficiencies than clay and silt with as high as $65 \%$ difference. Using the single acid for washing, inorganic acids are more efficient than organic acids; hydrochloric acid is more efficient than nitric acid. For "sand 1" and "sand 2" particles, the acid-washing method (by $0.1 \mathrm{M} \mathrm{HCl}$ or $\mathrm{HNO}_{3}$ ) can remove more than $90 \%$ cadmium while the efficiency is around only $50 \%$ for silt and $32 \%$ for clay. Figure 7 shows the cadmium removal efficiency using the hydrochloric/citric mixture. The results show that the mixed acid solution is $2.5 \%$ more effective than single acids in removing cadmium from soils of large particles.

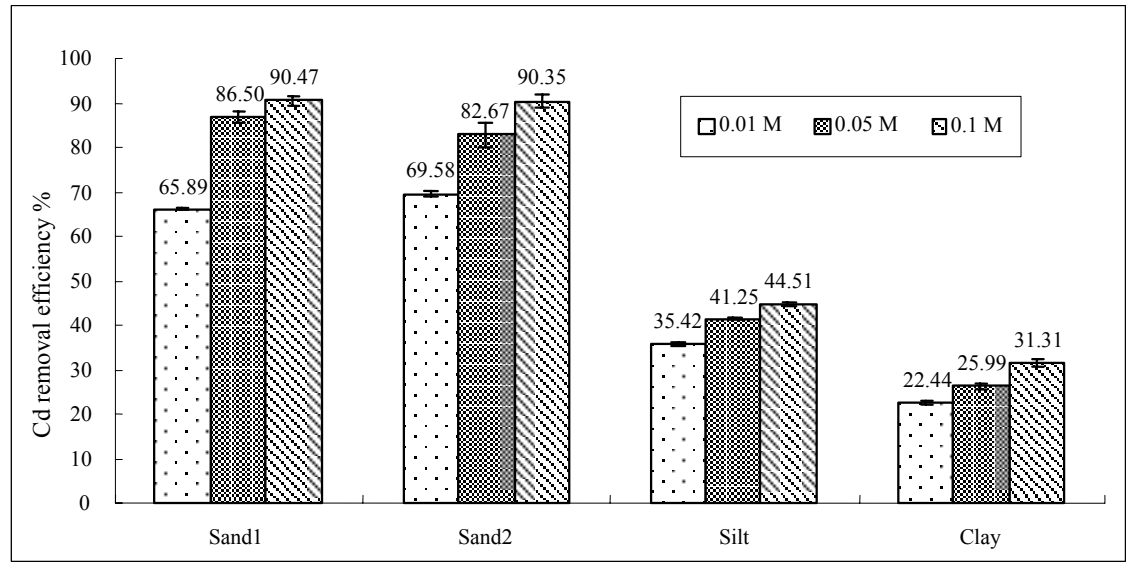

Figure 5: The metal Cd removal efficiencies from soil containing particles of various sizes using nitric acid.

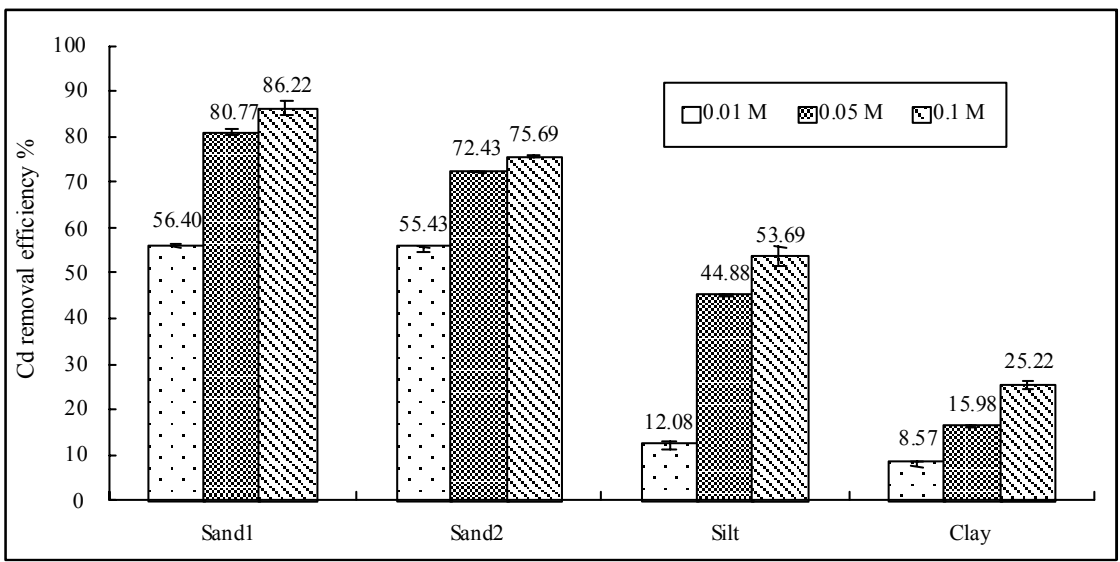

Figure 6: The metal Cd removal efficiencies from soil containing particles of various sizes using citric acid. 


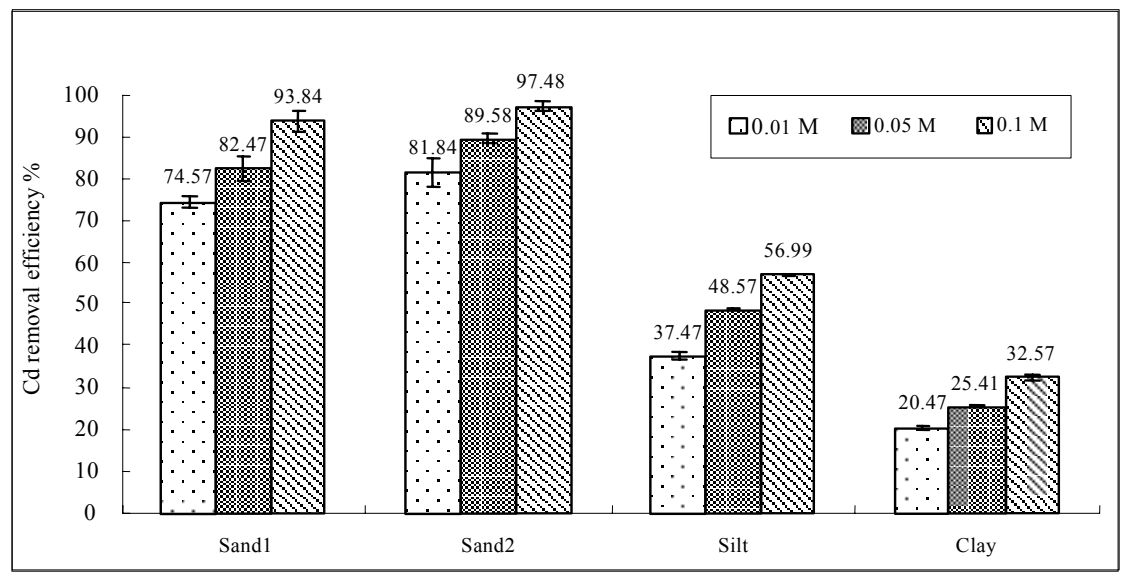

Figure 7: The metal $\mathrm{Cd}$ removal efficiencies from soil containing particles of various sizes using the hydrochloric/citric (1:1) mixture.

\section{Conclusions and recommendations}

Results obtained in this research show that the organic content, CEC and heavy metal content in the soil are somewhat consistent. Particles with diameters greater than $0.053 \mathrm{~mm}$ constitute more than $70 \%$ of the total soil mass but contain the lowest metal contents. They have the high metal removal efficiency and are easily separated from liquid. On the contrary, sol particles with diameters less than $0.053 \mathrm{~mm}$ contribute to $30 \%$ of the total soil mass especially the clay portion that is only $5.5 \%$ of the total mass. This portion of soil has the highest metal content and the lowest removal efficiency in addition to being more difficult to be separated by sedimentation from the liquid. The results obtained in this research indicate the use of a prior screening procedure to removal particles of small diameters that have the lowest acid-washing efficiencies. The acid-washing carried out on large particles will be efficient in removing heavy metal while the separated smaller particles can be treated using other treatment methods such that the overall treatment efficiency can be optimized.

\section{References}

[1] Evanko, C.R. \& Dzombak, D.A., Remediation of metals-contaminated soils and groundwater. Technology evaluation report, TE-97-01, Groundwater Remediation Technologies Analysis Center, 1997.

[2] Rosetti, P.K., Possible methods of washing fine soil particles contaminated with heavy metals and radionuclides. M.S. Thesis, Carnegie Mellon University, Pittsburgh, PA, 1993. 
114 Geo-Environment and Landscape Evolution II

[3] Anderson, R., Rasor, E. \& Ryn, F.V., Particle size separation via soil washing to obtain volume reduction. Journal of Hazardous Materials, 66, pp. 89-98, 1999.

[4] Sheets, R.G. \& Bergquist, B.A., Laboratory treatability testing of soils contaminated with lead and PCBs using particle-size separation and soil washing. Journal of Hazardous Materials, 66, pp. 137-150, 1999.

[5] Di Palma, L. \& Medici, F., Recovery of copper from contaminated soil by flusing. Waste Management, 22, pp. 883-886, 2002. 